\title{
Flexible and Adhesive SERS Active Tape for Rapid Detection of Pesticide Residues in Fruits and Vegetables
}

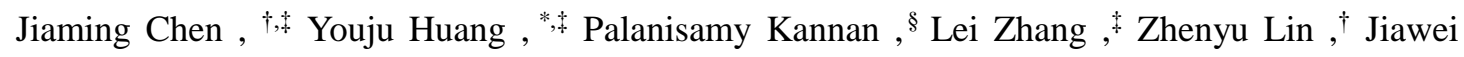
Zhang,+ Tao Chen $,{ }^{*}, *$ and Longhua Guo ${ }^{*}, \dagger$

$\dagger$ Institute of Nanomedicine and Nanobiosensing, Ministry of Education Key Laboratory of Analysis and Detection Technology for Food Safety, College of Chemistry, Fuzhou University, Fuzhou 350116, China

$\ddagger$ Division of Polymer and Composite Materials, Ningbo Institute of Material Technology and Engineering Chinese Academy of Sciences, No. 1219 Zhongguan West Road, Zhenhai District, Ningbo 315201, China

$\S$ Singapore Centre on Environmental Life Science Engineering, Nanyang Technological University,

Singapore 639798

\footnotetext{
*Corresponding Authors: E-mail: yjhuang@nimte.ac.cn; tao.chen@nimte.ac.cn; and guolh@fzu.edu.cn.
} 


\section{Table of Content}

1. Figure S1. SEM image of gold particles with the size of $25 \mathrm{~nm}$

2. Figure S2. SEM image of Au NPs decorated adhesive tape from large area

3. Figure S3. SERS spectra of 4-Mpy at different storage times

4. Figure S4. The SERS spectra of different concentrations of thiram and chlorpyrifos

5. Figure S5. Effect of the quantity of AuNPs that dropped on the tape to the intensity of Raman shift

6. Figure S6. Robustness of the SERS tape for real sample assay

7. Table S1. The Comparison of Maximum Residue Levels and SERS Limit of Detection of Three Pesticides on Cucumber 
1. Figure S1. SEM image of gold particles with the size of $25 \mathrm{~nm}$.

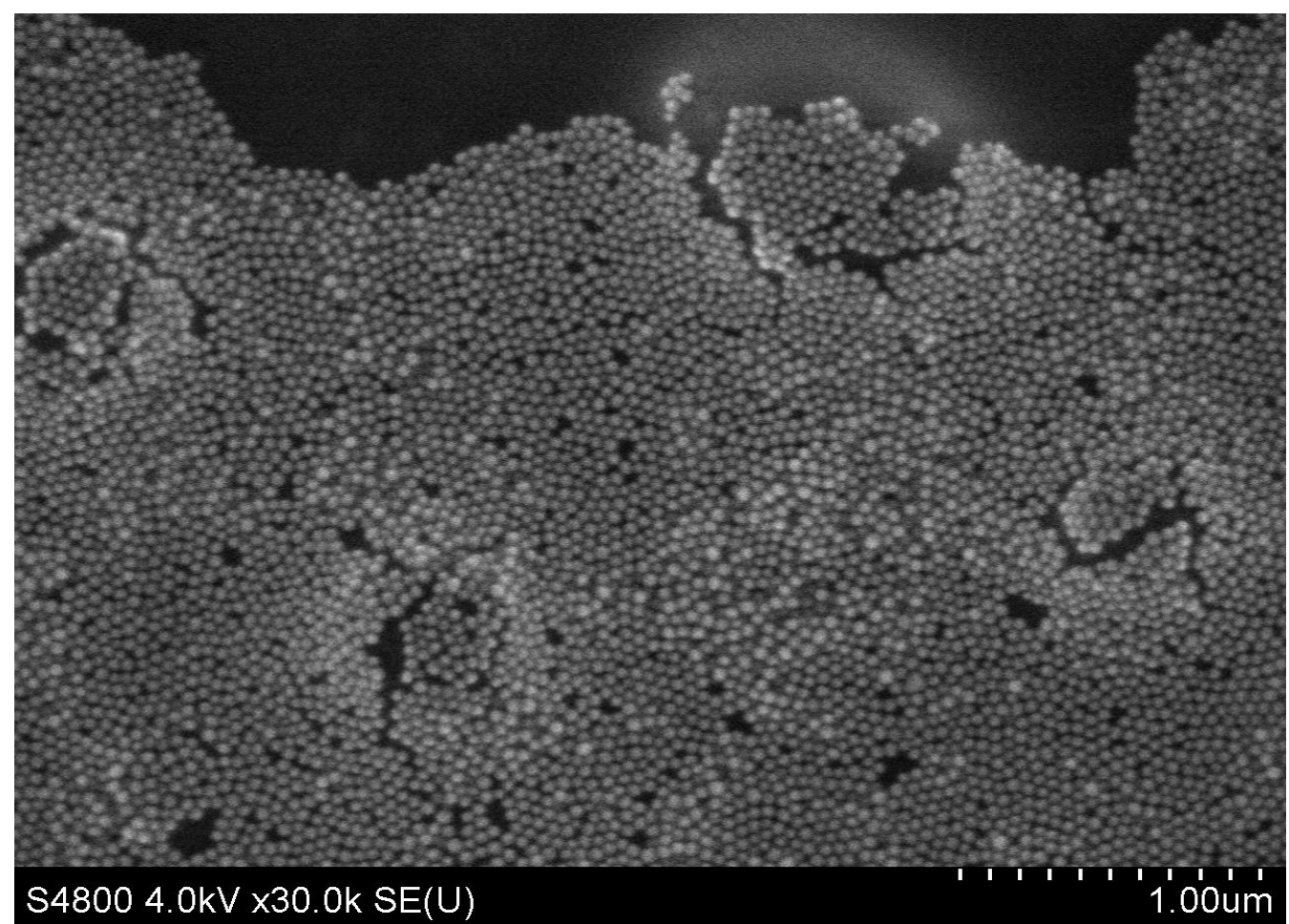


2. Figure S2. SEM image of Au NPs decorated adhesive tape from large area.

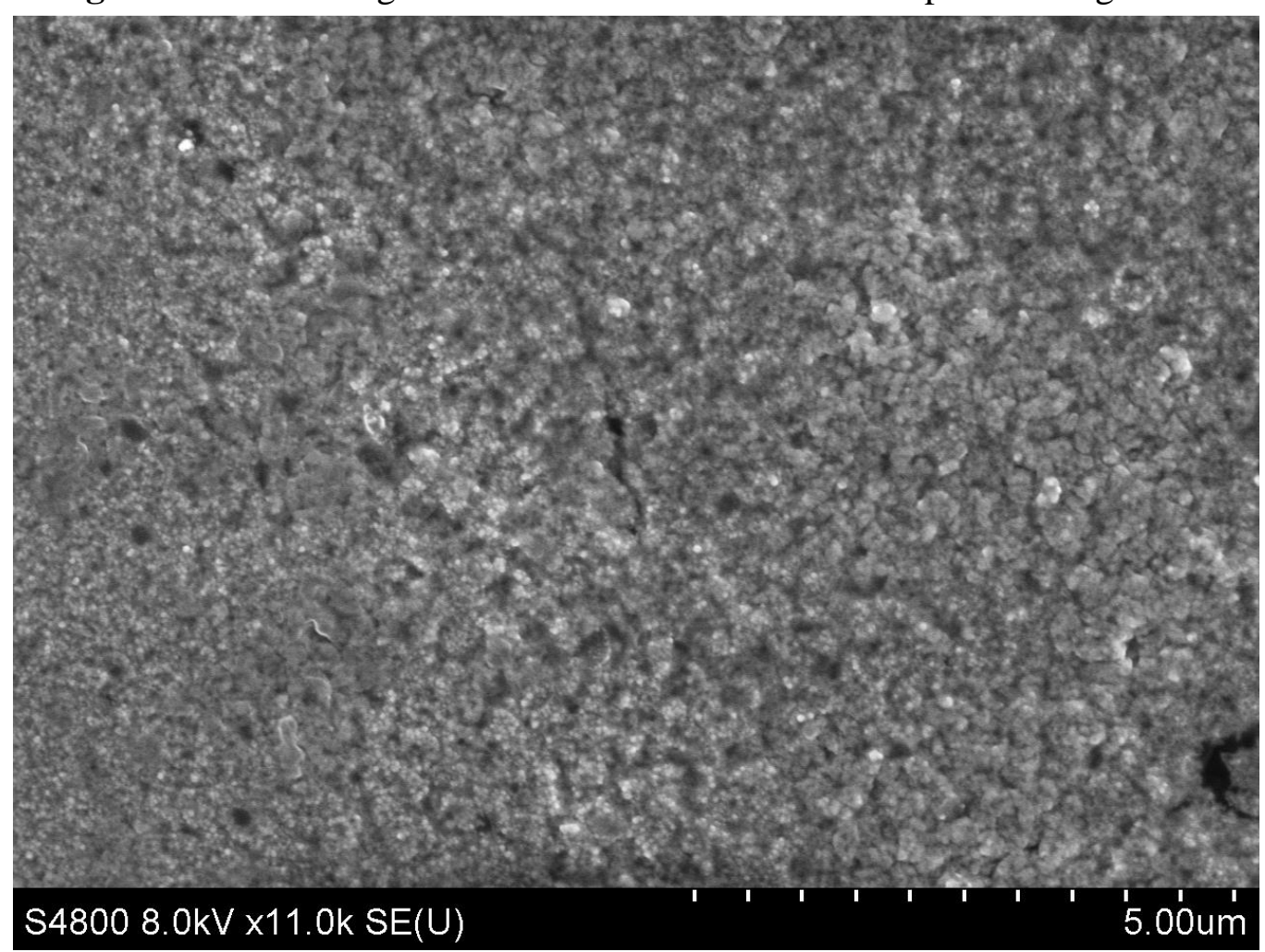


3. Figure S3. SERS spectra of 4-Mpy at different storage times

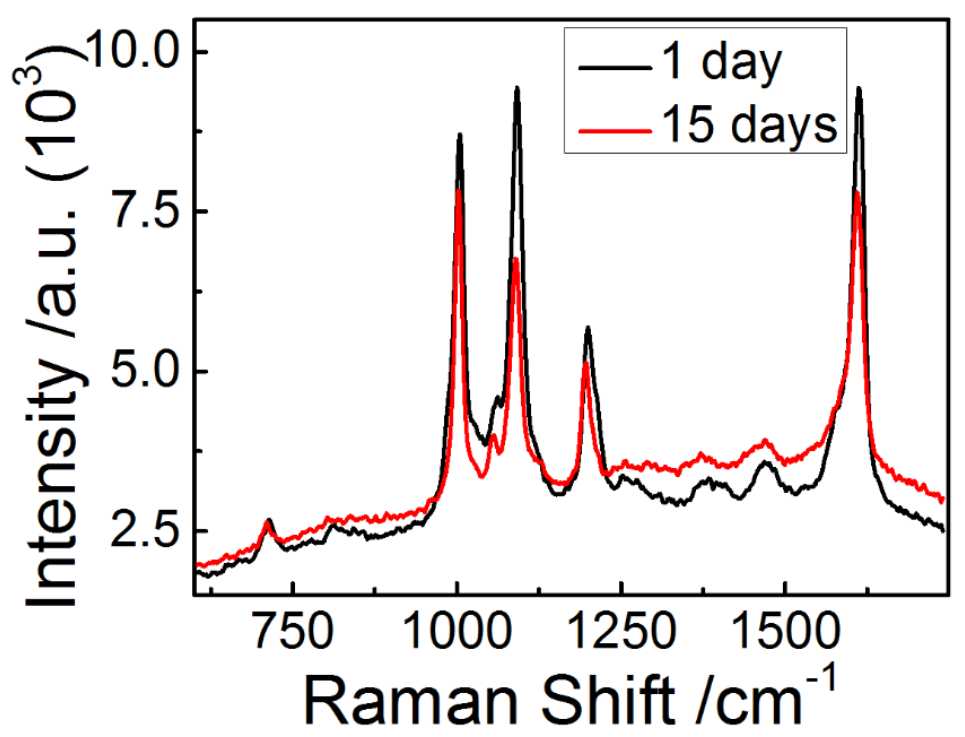

Figure S3. SERS spectra of 4-Mpy at different storage times: 1 day and 15 days. 10 $\mu \mathrm{L}$ of $10 \mu \mathrm{M} 4-\mathrm{Mpy}$ solution was used for each sample. Exposure time was $10 \mathrm{~s}$, laser wavelength was $633 \mathrm{~nm}$ and laser power was $1.7 \mathrm{~mW}$. 
4. Figure S4. The SERS spectra of different concentrations of thiram and chlorpyrifos
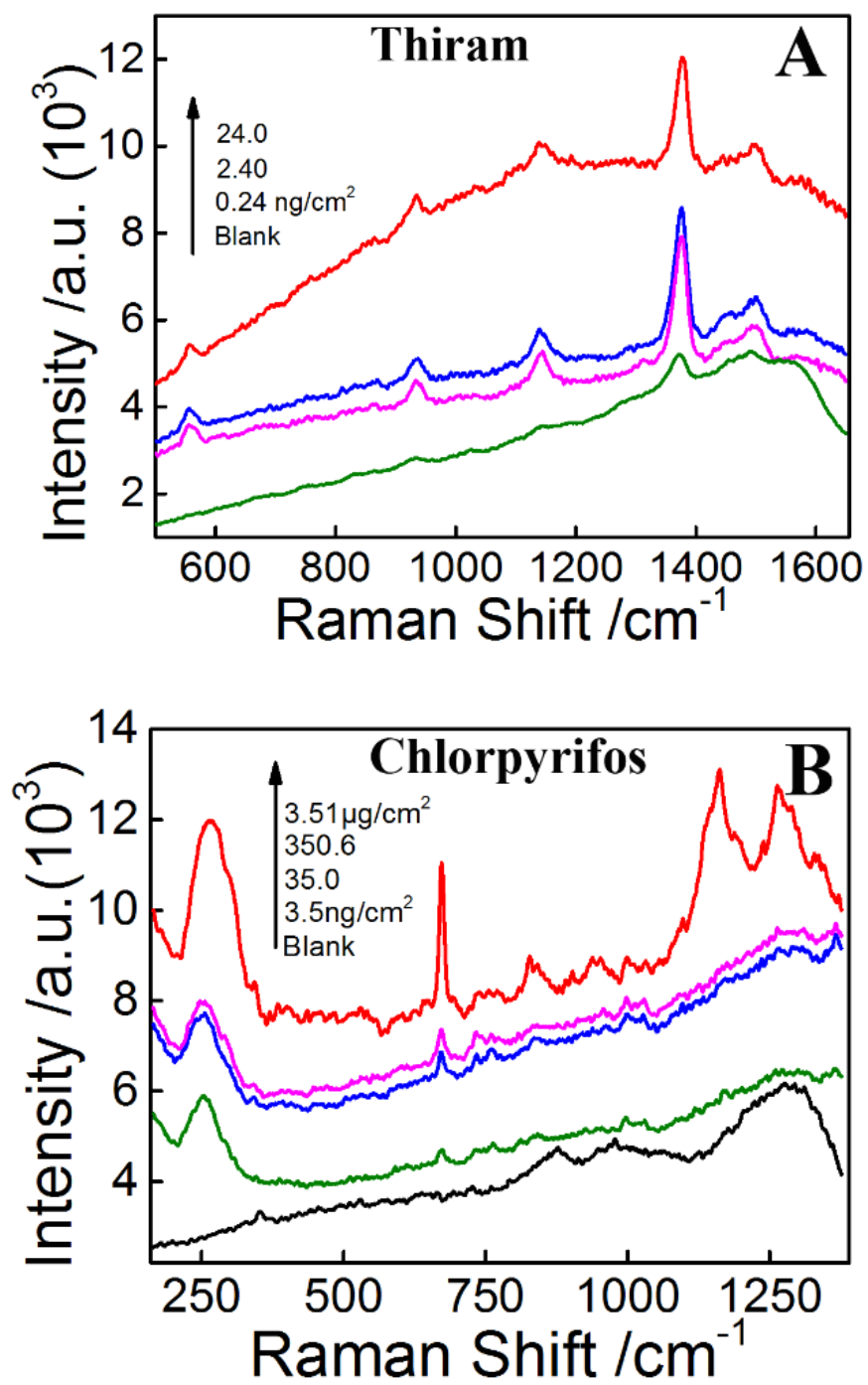

Figure S4. The SERS spectra of different concentrations of thiram (A) and chlorpyrifos (B). All these spectra were collected by SERS tape from cucumber peels. The exposure time was $10 \mathrm{~s}$, the laser wavelength was $633 \mathrm{~nm}$ and laser power was $1.7 \mathrm{~mW}$. 
5. Figure S5. Effect of the quantity of AuNPs that dropped on the tape to the intensity of Raman shift

Our investigation revealed that the amount of AuNPs loaded on the tape would influence both the Raman intensity and peak shape. We had optimized the concentration of $\mathrm{Au}$ NPs (from $5 \mathrm{mM}$ to $15 \mathrm{mM}$, calculated by the original concentration of $\mathrm{AuCl}_{4}^{-}$), and the results are shown in Figure S5 A (below). As shown in Figure S5 A, the peak position of the main Raman shifts was almost unchanged. However, the intensity and shape of peaks had changed with some special Raman peak rising significantly. These shape-changed Raman shifts, which located at 1007 $\mathrm{cm}^{-1}$ and $1576 \mathrm{~cm}^{-1}$, are assigned to the ring breathing mode and $(v) \mathrm{C}=\mathrm{C}$ with deprotonated nitrogen from 4-Mpy (Guo, H.; Ding, L.; Mo, Y. J. Mol. Struct. 2011, 991, 103-107). It suggested that more 4-Mpy molecule were attached to Au NPs when more AuNPs are loaded onto the tap.

On the other hand, although larger concentration of Au NPs would generate greater SERS signal, the reproducibility of Raman signals was poor in case of a high amount of Au NPs loaded onto the tap (please see the error bars in Figure S5 B). This kind of poor reproducibility indicated the uniformity of AuNP loaded tap is poor when high concentration of AuNPs were used. In order to obtain a compromise between the intensity of SERS signal and reproducibility, $5 \mathrm{mM}$ of Au NPs was used in our experiments.
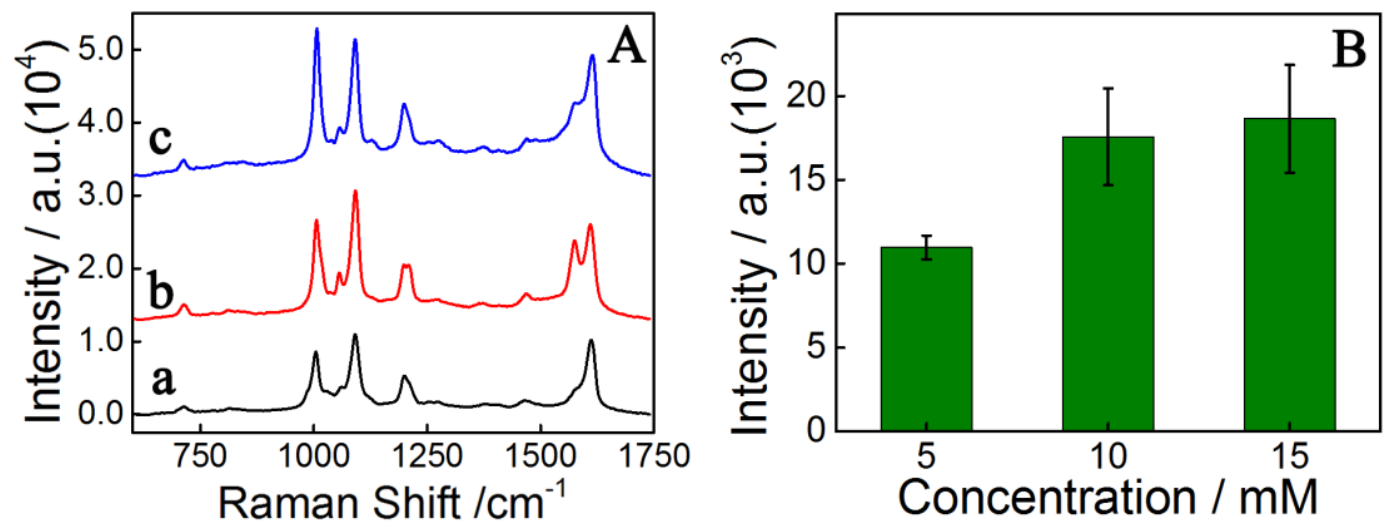

Figure. S5 SERS spectra of 4-Mpy (A) on different concentration of AuNPs coated adhesive tape. a: $5 \mathrm{mM}$; b: $10 \mathrm{mM}$; c: $15 \mathrm{mM}$. The statistics of Raman shift intensities (B) at $1098 \mathrm{~cm}^{-1}$ related to the concentration of Au NPs. 
6. Figure S6 Robustness of the SERS tape for real sample assay

To test the solubility of polymer substrate and the strength of binding between $\mathrm{Au}$ NPs and the polymer layer, the prepared SERS tape was immersed into a glass dish containing $1 \mathrm{~mL}$ water for $24 \mathrm{~h}$, and then dried in the air. Figure S6-2 A, B and C show the photographs of the SERS tape before immersing, the wet SERS tape and the immersed tape after dried. It can be seen that the macroscopic pattern of the AuNP layer is almost the same before and after immersed in water. It indicates that most of the Au NPs are still attached on the tape after immersing. The SEM images also show that no significant density variations of AuNPs are observed before and after immersing (Figure S6-2 D and E).

To further confirm our deduction, we tested the absorption spectra of the solution which previously used for immersing the SERS tape, and the result was compared with those of sample blank and the same amount $(10 \mu \mathrm{L} 5 \mathrm{mM}$ prepared Au NPs diluted to $1 \mathrm{~mL}$ with water) of Au NP disposed in solution (Figure S6-2 F). It can be clearly seen that only slight absorption peak at $520 \mathrm{~nm}$ is observed as compared with that of the sample blank. Compared with the spectrum of the same amount of AuNP disposed in water (the purple curve in Figure S6-2 F), this small peak is negligible. These results indicate that only small amount of AuNPs are detached from the substrate after immersing the SERS tape in water for $24 \mathrm{~h}$. It is worth to note that in actual sample analysis, only small amount of sample solution would be deposited on the SERS substrate, and the solution would be dried within a few minutes. Therefore, we conclude that the proposed SERS tape is robust enough for real sample assay.
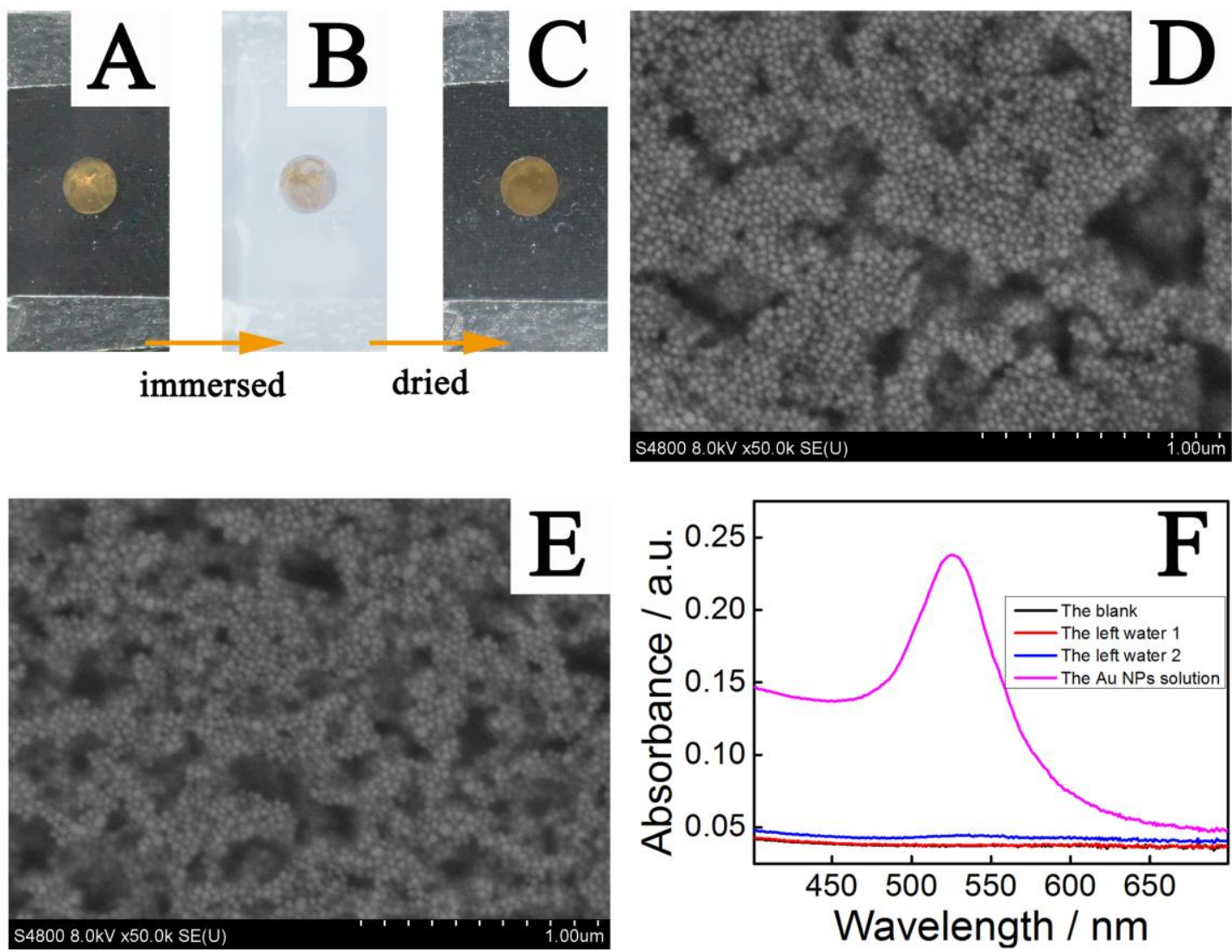
Figure.S6 The photographs of the prepared SERS tape (A), the immersed SERS tape (B), the dried SERS tape (C). The SEM image of the prepared SERS tape (D) the dried SERS tape (E). (F) The absorption spectra of the blank (water), the left water1 (after adhesive tape immersing), the left water 2 (after SERS tape immersing) and the Au NPs solution ( $10 \mu \mathrm{L} 5 \mathrm{mM}$ prepared Au NPs / 1mL water). 
7. Table S1. The Comparison of Maximum Residue Levels and SERS Limit of Detection of Three Pesticides on Cucumber

\begin{tabular}{cccc}
\hline & parathion-methyl & thiram & chlorpyrifos \\
\hline MRLs (GB 2763-2014 ) & $0.02 \mathrm{mg} / \mathrm{kg}$ & $5 \mathrm{mg} / \mathrm{kg}$ & $0.1 \mathrm{mg} / \mathrm{kg}$ \\
MRLs (EC NO 396/2005) & $0.01 \mathrm{mg} / \mathrm{kg}$ & $0.1 \mathrm{mg} / \mathrm{kg}$ & $0.05 \mathrm{mg} / \mathrm{kg}$ \\
SERS limit of detection ${ }^{\mathrm{c}}$ & $0.002 \mathrm{mg} / \mathrm{kg}$ & $0.0002 \mathrm{mg} / \mathrm{kg}$ & $0.003 \mathrm{mg} / \mathrm{kg}$ \\
\hline
\end{tabular}

a MRLs is National food safety standard (GB 2763-2014) in China.

b MRLs is Regulation (EC) No 396/200 in European Union.

c The concentration converted from mass-to-area ratio to mg per kilogram, was roughly calculated according to previous work (Anal. Chem. 2012, 84 (1), 255-61). 